\title{
SUPPORTING INFORMATION \\ PEGylated Artificial Antibodies: Plasmonic Biosensors with Improved Selectivity
}

\author{
Jingyi Luan ${ }^{1}$, Keng-Ku Liu ${ }^{1}$, Sirimuvva Tadepalli ${ }^{1}$, Qisheng Jiang ${ }^{1}$, Jeremiah J. Morrissey ${ }^{2,3}$, \\ Evan D. Kharasch ${ }^{2,3,4,5}$ and Srikanth Singamaneni ${ }^{1,3 *}$ \\ ${ }^{1}$ Department of Mechanical Engineering and Materials Science, Institute of Materials Science \\ and Engineering, Washington University in St. Louis, St Louis, MO, 63130, USA. \\ ${ }^{2}$ Department of Anesthesiology, Division of Clinical and Translational Research, Washington \\ University in St. Louis, St. Louis, MO, 63110, USA \\ ${ }^{3}$ Siteman Cancer Center, ${ }^{4}$ Department of Biochemistry and Molecular Biophysics, Washington \\ University in St. Louis, St. Louis, MO, 63110, USA. ${ }^{5}$ The Center for Clinical Pharmacology, St. \\ Louis College of Pharmacy and Washington University School of Medicine, St. Louis, MO, USA
}

*To whom correspondence should be addressed: singamaneni@wustl.edu 


\section{Figures}
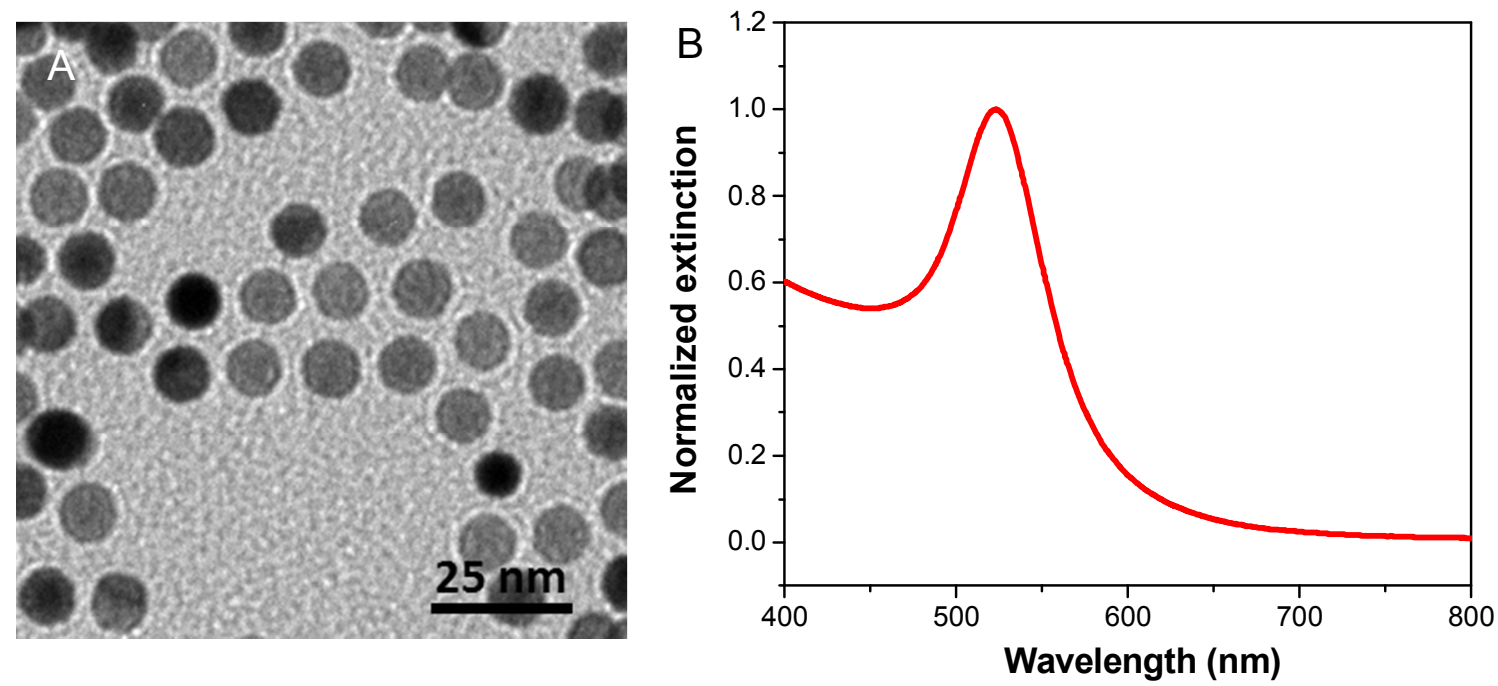

Figure S1. (A) TEM image of Au nanospheres (AuNS) employed as seeds for the synthesis of $\mathrm{Ag} @$ Au nanocubes (B) Vis-NIR extinction spectrum of Au nanospheres. 


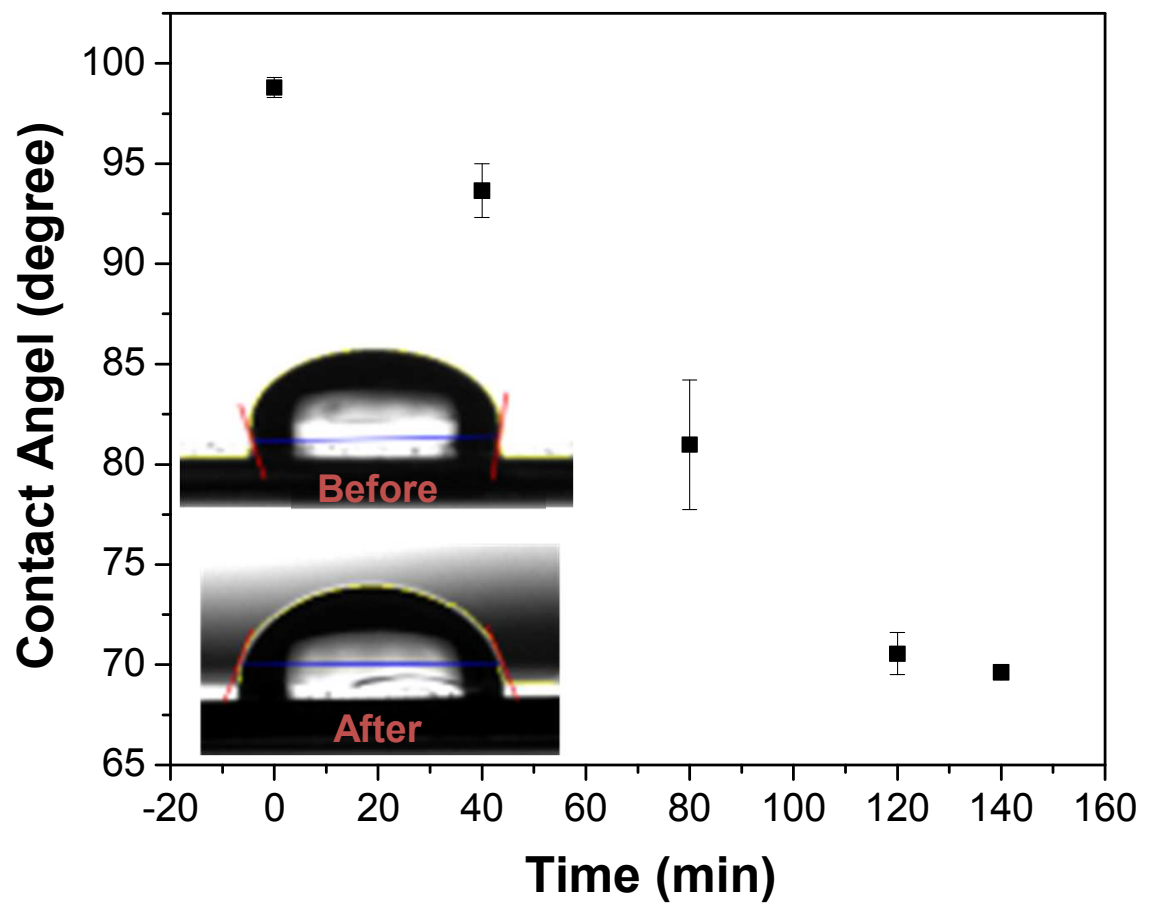

Figure S2. Contact angle vs. PEGylation time showing the progressive decrease in the contact angle of the MIP (inset shows the water drop on the MIP surface before and after PEGylation for 140 min) 


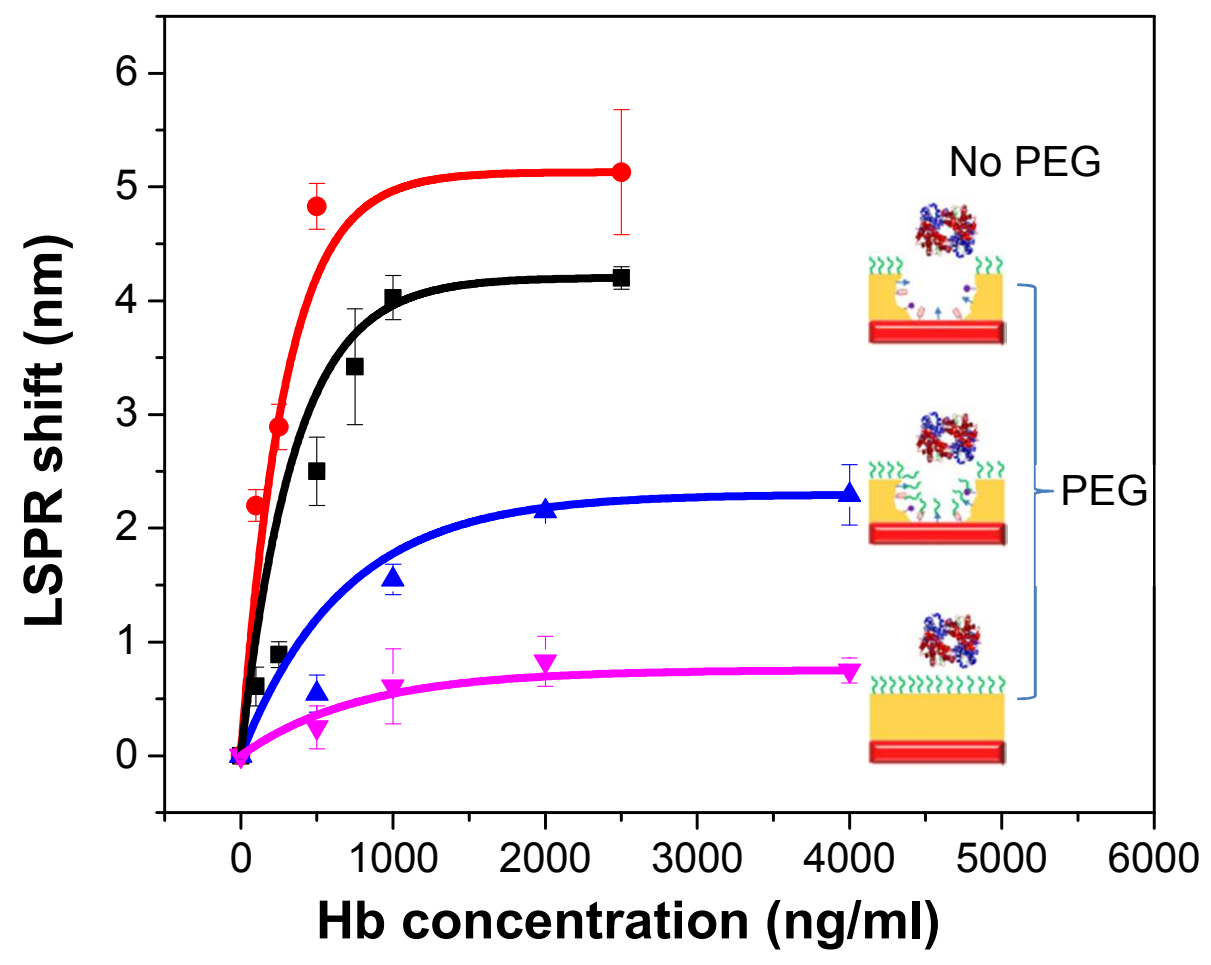

Figure S3. LSPR shift vs. concentration of the target biomarker for non-PEGylated MIP (red), normally PEGylated MIP (black), cavity PEGylated MIP (blue) and non-cavity PEGylated MIP (pink) 


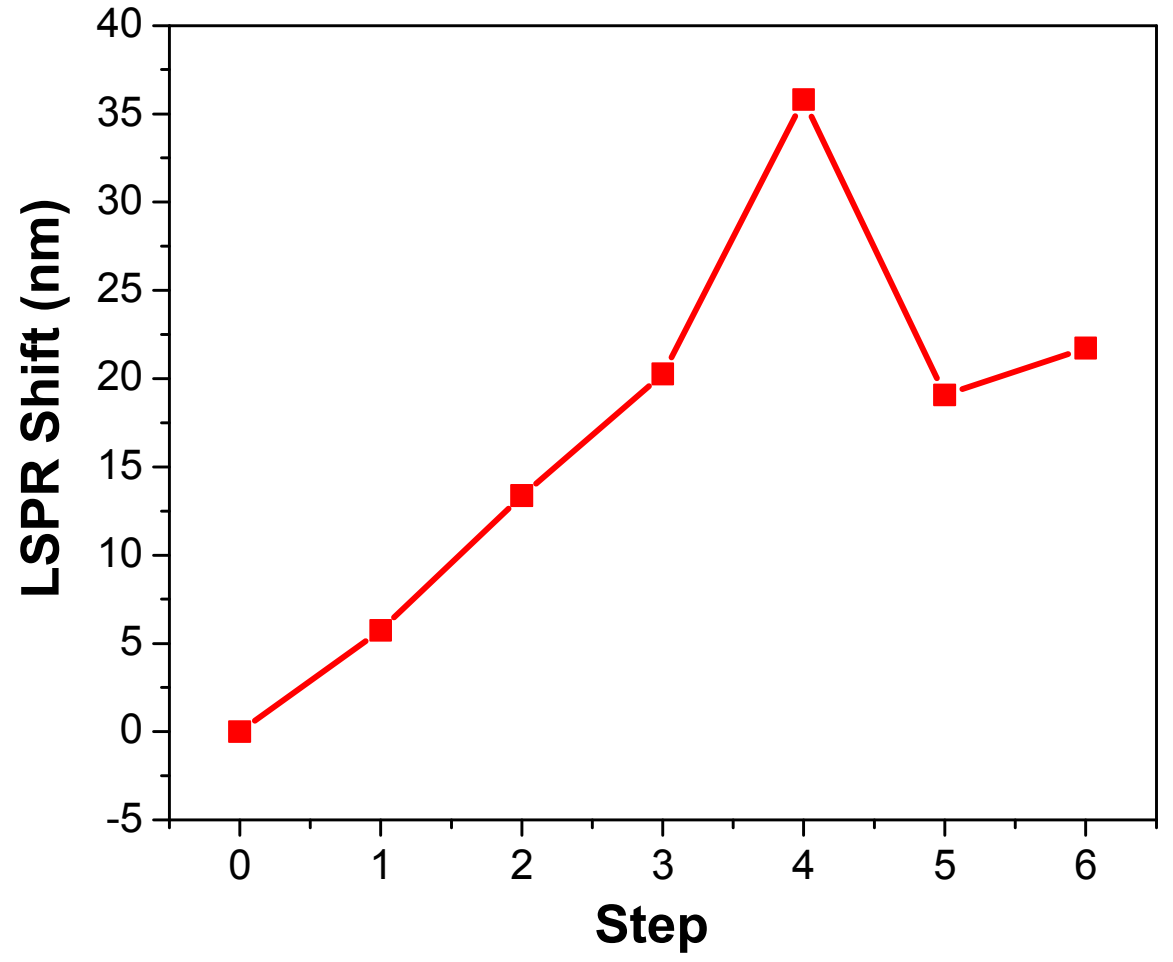

Figure S4. LSPR shift of each steps for modifying protein blocked artificial antibody biosensor. 